\title{
MASTEP \\ THE SET CODES - IBM 704 \\ CODES FOR THE CALCULATION \\ OF THE STRESSES IN A \\ PRESSURE VESSEL WITH AN \\ ELLIPSOIDAL HEAD
}

JUNE 1959

CONTRACT AT-11-1-GEN-14

BETTIS PLANT-PITTSBURGH, PA. OPERATED FOR THE U.S. ATOMIC ENERGY COMMISSION BY BETTIS ATOMIC POWER DIVISION, WESTINGHOUSE ELECTRIC CORPORATION 


\section{DISCLAIMER}

This report was prepared as an account of work sponsored by an agency of the United States Government. Neither the United States Government nor any agency Thereof, nor any of their employees, makes any warranty, express or implied, or assumes any legal liability or responsibility for the accuracy, completeness, or usefulness of any information, apparatus, product, or process disclosed, or represents that its use would not infringe privately owned rights. Reference herein to any specific commercial product, process, or service by trade name, trademark, manufacturer, or otherwise does not necessarily constitute or imply its endorsement, recommendation, or favoring by the United States Government or any agency thereof. The views and opinions of authors expressed herein do not necessarily state or reflect those of the United States Government or any agency thereof. 


\section{DISCLAIMER}

Portions of this document may be illegible in electronic image products. Images are produced from the best available original document. 


\title{
THE SET CODES - IBM 704 CODES FOR THE CALCULATION OF THE STRESSES IN A PRESSURE VESSEL WITH AN ELLIPSOIDAL HEAD G.G. Bilodeau - J.B. Callaghan - H. Kraus
}

Contract AT-11-1-GEN-14

June 1959

Price $\$ 1.00$

Available from the Office of Technical Services,

Department of Commerce,

Washington 25, D. C.

\section{NOTE}

This document is an interim memorandum prepared primarily for internal reference and does not represent a final expression of the opinion of Westinghouse. When this memorandum is distributed externally, it is with the express understanding that Westinghouse makes no representation as to completeness, accuracy, or usability of information contained therein.

\author{
BETTIS PLANT $\bullet$ PITTSBURGH, PA. \\ OPERATED FOR THE U.S. ATOMIC ENERGY COMMISSION BY \\ BETTIS ATOMIC POWER DIVISION, WESTINGHOUSE ELECTRIC CORPORATION
}




\section{SPECIAL EXXERNAL DISTRIBUUTION}

Manager, Pittsburgh Naval Reactors Operations Office, AEC

Argonne National Laboratory, W. F. Miller Brookhaven National Laboratory, J. Chernick Brookhaven National Laboratory, M. Rose David Taylor Model Basin, H. Polachek

Knolls Atomic Power Laboratory, R. Ehrlich

Los Alamos Scientific Laboratory, B. Carison

New York University, R. Richtmyer

Oak Ridge National Laboratory, A. Householder

University of California Radiation Laboratory, Livermore, S. Fernbach

Th/s report was prepared as an account of Government sponsored work. Neither the United states, nor the Commission, nor any person acting on behalf of the Commission:

A. Makes any warranty or representation, expressed or implied, with respect to the accuracy, completeness, or usefulness of the information contained in this report, or that the use of any information, apparatus, method, or.process disclosed in this report may not infringe privately owned rights; or

B. Assumes any liabilities with respect to the use of, or for damages resulting from the use of any information, apparatus, method, or process disclosed in this report.

As used in the above, "person acting on behalf of the Commission" includes any employe or contractor of the Commission, or employe of such contractor, to the extent that such employe or contractor of the Commission, or employe of such contractor prepares, disseminates, or provides access to, any information pursuant to his employment or contract with tlie Commlssion, or his employiment with such contractor. 


\section{CONTENTS}

THEORY

Page No.

SOLUTION OF THE ELLIPSOIDAL SHELL EQUATIONS

Boundary Conditions

DIFFERENCE EQUATIONS

Case I: $\theta_{n}$ an Interior Point

Case II: $\mathrm{n}=\mathrm{N}$

1

GENERAL METHODS OF SOLUTION

Method (A): The SET02 Code

Method (B): The SET03 Code

CALCULATION OF THE STRESSES

INPUT PREPARATION

OUTPUT DESCRIPTION

CONCLUSIONS

OPERATING INSTR UCTIONS

Code Restrictions

APPENDIX I: SAMPLE PROBLEM

ACKNOWLEDGMENT

REFERENCES 


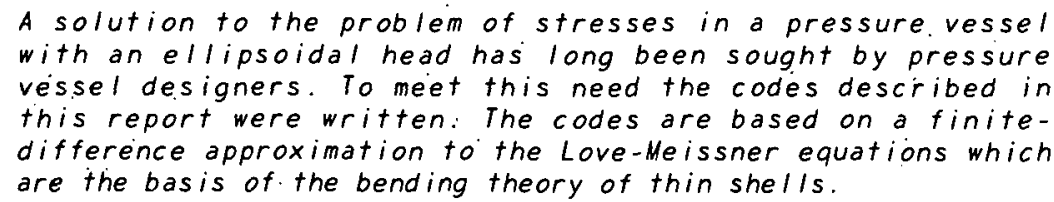

THE SET CODES-IBM 704 CODES FOR THE

CALCULATION OF THE STRESSES IN A PRESSURE

VESSEL WITH AN ELLIPSOIDAL HEAD

G. G. Bilodeau, J. B. Callaghan, and H. Kraus

A means of determining the stresses in an ellipsoidal pressure vessel head has long been * sought by pressure vessel designers. In the nuclear power plant industry, for example, the ellipsoidal shell appears frequently as the head of a steam generator or as the bottom of a reactor pressure vessel. In such applications there are discontinuity stresses set up because of the difference in the expansion experienced by the ellipsoidal shell and the cylindrical shell to which it is attached when the entire assembly is loaded by internal pressure. At present there is no method available for the determination of these.stresses, and designers have been forced to make overly conservative assumptions to overcome this deficiency.

The basic differential equations of the problem have been available for many years, although their complexity has not permitted a convenient analytical solution. It was believed, however, that a high-speed electronic computing machine would be ideally suited for the solution of these equations. Hence, the IBM 704 codes described in this report were written.

\section{THEORY}

The configuration which will be considered in this discussion consists of an ellipsoid of revolution mounted on a long cylinder, as shown in Fig. 1. * The method of analysis generally applied to this type of shell is referred to as the bending theory of thin shells. A shell is considered thin when its diameter is more than ten times its thickness. This assumption allows analytical solutions to be found for many shells, although, for the ellipsoidal shell, analytical solutions have not been found in spite of this assumption. An exact theory for treating thick shells is not available at the present time.

* Note that the middle surfaces of the head and cylinder must coincide. 


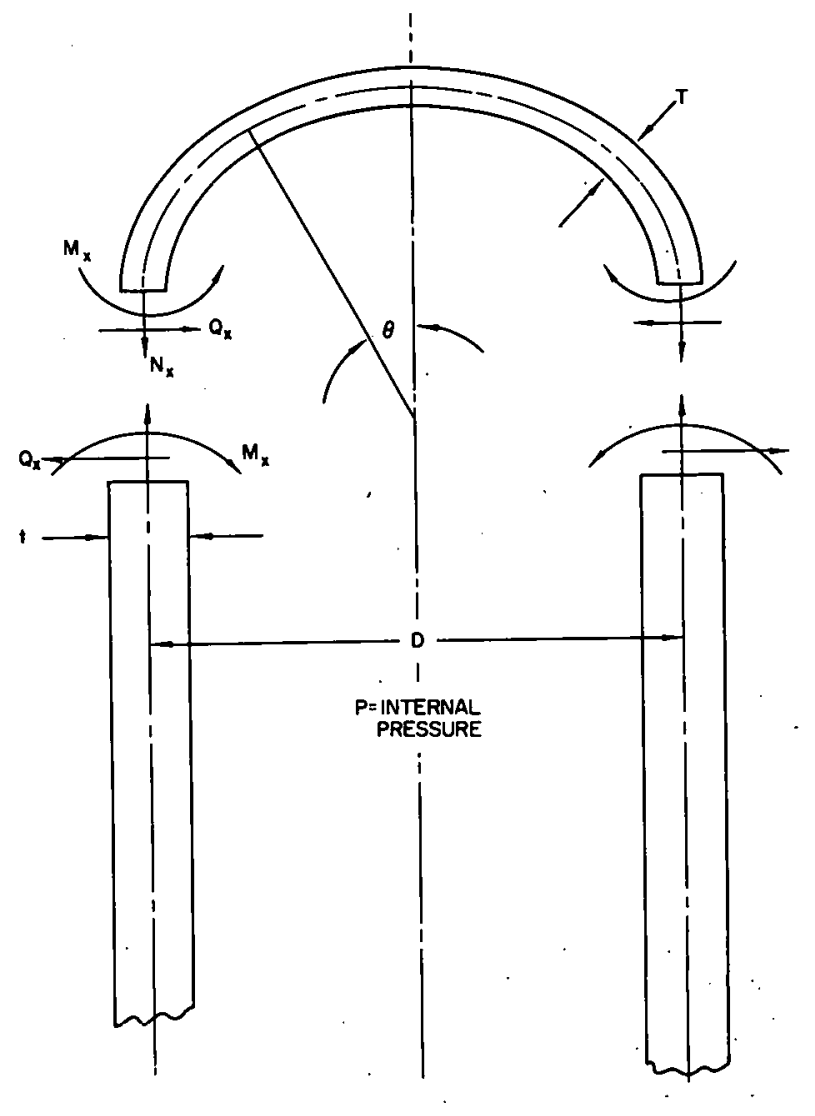

Fig. I Ellipsoidal Shell Mounted on a Cylinder Showing Loads at Junction

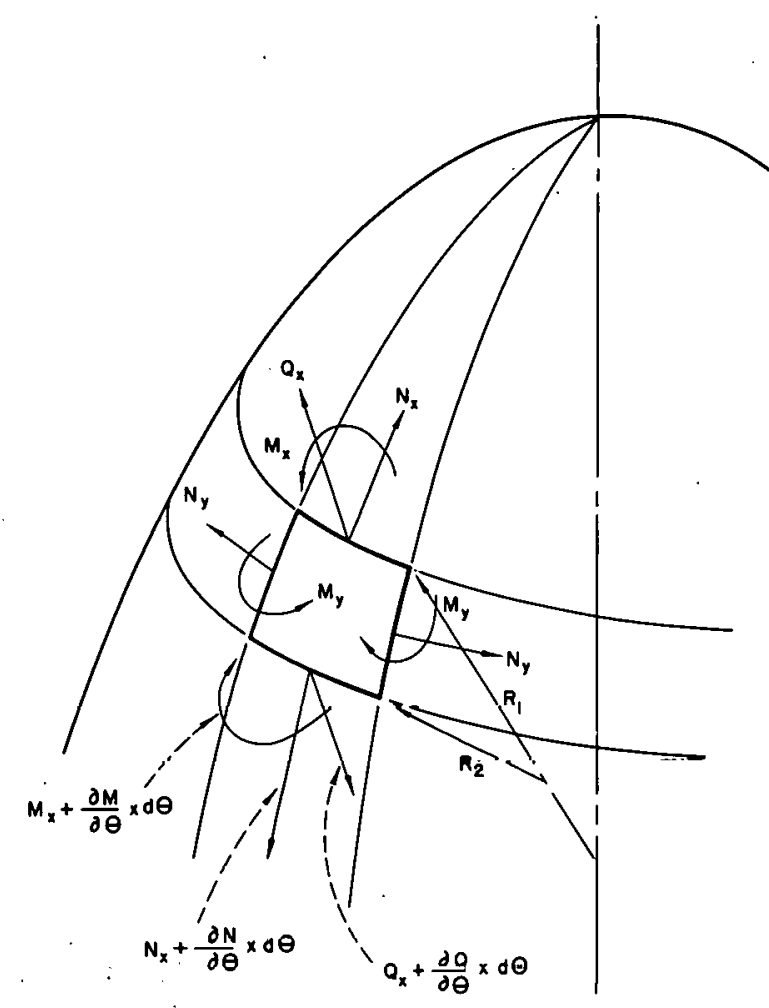

Fig. 2 A Differential Element in a Shell of Revolution Showing the Movements and rories Acting on II (from Ref 2)

The bending theory of thin shells is very well described by Watts and Burrows (Ref 1) and by Timoshenko (Ref 2). The method presented in this report will draw largely upon the Watts and Burrows paper.

The analysis of any shell of revolution begins with the consideration of the forces and moments acting on a differential element of the shell as shown in Fig. 2. A summation of the forces and of the moments then yields three equations of equilibrium for a differential element in a shell under internal pressure:*

$$
\begin{aligned}
& \underset{d \theta}{d}\left[N_{x} R_{2} \sin \theta\right]-N_{y} R_{1} \cos \theta+Q_{x} R_{2} \sin \theta=0 \\
& \frac{d}{d \theta}\left[Q_{x} R_{2} \sin \theta\right]-N_{y} R_{1} \sin \theta-N_{x} R_{2} \sin \theta=-P R_{1} R_{2} \sin \theta
\end{aligned}
$$

and

$$
\frac{\mathrm{d}}{\mathrm{d} \theta}\left[\mathrm{M}_{\mathrm{x}} \mathrm{R}_{2} \sin \theta\right]-M_{y} R_{1} \cos \theta-\mathrm{Q}_{\mathrm{x}} \mathrm{R}_{2} \mathrm{R}_{1} \sin \theta=0
$$

where

$\theta=$ angle between the axis of revolution and a perpendicular to the middle surface of the shell at any point

*Reference 1 
$R_{1}=$ radius at any point of a middle surface section cut by a plane through the axis of revolution

$R_{2}=$ distance measured along a normal to any point on the middle surface, between the middle surface and the axis of revolution

$\mathrm{P}=$ internal pressure acting in the shell

$\mathrm{N}_{\mathrm{x}}=$ axial membrane force

$\mathrm{N}_{\mathrm{y}}=$ circumferential membrane force

$\mathrm{M}_{\mathrm{X}}=$ axial bending moment

$\mathrm{M}_{\mathrm{y}}=$ circumferential bending moment

$\mathrm{Q}_{\mathrm{x}}=$ shear force acting along a normal to the middle surface.

In Eqs (1), (2), and (3) there are five unknown quantities which are $N_{x}, N_{y}, M_{x}, M_{y}$, and $Q_{x}$. The first four quantities can be expressed in terms of the rotation and the deflection of the differential element (Ref 1) as follows:

$$
\begin{gathered}
N_{x}=\frac{E T}{R_{1}\left(1-\mu^{2}\right)}\left[\left(\frac{d u}{d \theta}+w\right)+\mu\left(\frac{R_{1}}{R_{2}}\right)(w+u \cot \theta)\right], \\
N_{y}=\frac{E T}{R_{1}\left(1-\mu^{2}\right)}\left[\mu\left(\frac{d u}{d \theta}+w\right)+\frac{R_{1}}{R_{2}}(w+u \cot \theta)\right], \\
M_{x}=\frac{E T^{3}}{m^{4} R_{1}}\left[\frac{d w}{d \theta}+\mu\left(\frac{R_{1}}{R_{2}}\right) w \cot \theta\right],
\end{gathered}
$$

and

$$
M_{y}=\frac{E T^{3}}{m^{4} R_{1}}\left[\mu\left(\frac{d W}{d \theta}\right)+\frac{R_{1}}{R_{2}} W \cot \theta\right]
$$

where

$\mathrm{T}=$ thickness of the ellipsoidal shell

$\mathrm{E}=$ Young's modulus

$\mu \quad=$ Poisson's ratio $^{\prime}$

$\mathrm{m}^{4}=12\left(1-\mu^{2}\right)$

$\mathrm{W}$ = rotation of a tangent to the middle surface, lying in a plane through the axis of revolution during deformation of the shell;

$\mathrm{W}=\frac{1}{\mathrm{R}_{1}}(\mathrm{u}-\mathrm{d} \mathrm{w} / \mathrm{d} \theta)$

$\mathrm{u}=$ deformation of any point on the middle surface measured along a tangent to the undeformed middle surface at the point and lying in a plane through the axis of revolution

$\mathrm{w}=$ deformation of any point on the middle. surface measured along a normal to the undeformed middle surface at the point and lying in a plane through the axis of revolution

When the expressions (4), (5), (6), and (7) are substituted into Eqs (1), (2), and (3), the resulting expressions can be simplified to give the following differential equations (known as the Iove-Meissner equations) relating the rotation and the shear: 


$$
L[V]+\frac{\mu}{R_{1}} V=-E T W+\Phi(\theta)
$$

and

$$
L[W]-\frac{\mu}{R_{1}} W=+\frac{V m^{4}}{E T^{3}} \text {. }
$$

In the se equations, $V=R_{2} Q_{x}, \Phi(\theta)$ is a function of load and shape, and L [ ] represents a differential operator which is defined as follows:

$$
L[\ldots]=\frac{R_{2}}{R_{1}^{2}} \frac{d^{2}(\ldots)}{d \theta^{2}}+\frac{1}{R_{1}}\left[\frac{d}{d \theta}\left(\frac{R_{2}}{R_{1}}\right)+\frac{R_{2}}{R_{1}} \cot \theta\right] \frac{d(\ldots)}{d \theta}-\frac{\cot ^{2} \theta}{R_{2}}(\ldots) .
$$

Up to this point the type of shell has not been specified. From the equations which have been presented it can be seen that, in order to make them apply to a particular shell, only a substitution of the appropriate expressions for the two radii of curvature $R_{1}$ and $R_{2}$ is needed.

Thus, for the pllipsinidal sherll,

$$
R_{1}=0.5 \mathrm{DBv}^{3}
$$

and

$$
\mathrm{R}_{2}=0.5 \mathrm{DBv} \text {, }
$$

where $\mathrm{D}=$ the major axis of the ellipse and is also the diameter of the cylinder, $\beta=$ ratio of the major axis of the ellipse to the minor axis of the ellipse, and $\left(\frac{1}{v}\right)=\left[1+\left(\beta^{2}-1\right) \sin ^{2} \theta\right]$.

For the cylindrical shell,

$$
\mathbf{R}_{1}=\infty
$$

and

$$
R_{2}=0.5 \mathrm{D}
$$

After substitution of Eqs (11) and (12) into Eqs (4) through (10), the following equations (Ref 1) are obtained for the ellipsoidal shell:

$$
\begin{aligned}
& M_{x}=\frac{\frac{2 E T^{3}}{4}}{m^{4} D \delta}\left[\frac{1}{v^{2}} \frac{d W}{d \theta}, \mu W \cot 0\right] \text {, } \\
& M_{y}=\frac{2 E T^{3}}{m^{4} D \delta}\left[\frac{\mu}{v^{2}} \frac{d W}{d \theta}+W \text { iul } \theta\right] \text {, } \\
& \mathrm{N}_{\mathrm{x}}-\frac{2 \mathrm{~V}}{\delta \mathrm{D}} \text { oot } \theta+0.35 \mathrm{PD} 6 \\
& N_{y}=\frac{2}{D \delta^{\prime}} \frac{d V}{d \theta}+0.25 P D\left[\frac{2 \delta^{2}-\beta^{2}}{\delta}\right] \text {, } \\
& L[V]+\mu V+0.5 \frac{D E T}{\delta^{\prime}} W=\frac{P D^{2} \beta^{2}}{8}\left(3 v^{2}+1\right)\left(1-v^{2}\right) \cot \theta \\
& L[\dot{W}]-\mu W-\frac{n}{2} \frac{\delta m^{4}}{E T^{3}} V=0 \text {, }
\end{aligned}
$$


and the differential operator for the ellipse appears as

$$
v^{2} L[\ldots]=\frac{d^{2}(\ldots)}{d \theta^{2}}+\left[\left(3-2 v^{2}\right) \cot \theta\right] \frac{d(\ldots)}{d \theta}-(. .)^{4} \cot ^{2} \theta \text {, }
$$

where $\delta=\beta v$ and $\delta^{\prime}=\beta v^{3}$

Equations (19) and (20) are the equations which have caused so much difficulty for analysts seeking solutions to ellipsoidal shell problems. These equations must be solved for $\mathrm{V}$ and $\mathrm{W}$ which are used to determine $M_{x}, M_{y}, N_{x}$, and $N_{y}$. The latter quantities are combined by using the following relations to obtain the stresses:

$$
\sigma_{x}=\frac{N_{x}}{T} \pm \frac{6 M_{x}}{T^{2}}
$$

and

$$
\sigma_{y}=\frac{N_{y}}{T} \pm \frac{6 M_{y}}{T^{2}}
$$

where $\sigma_{\mathrm{x}}=$ axial stress, $\sigma_{\mathrm{y}}=$ circumferential stress, and the positive sign is used for stresses on the outer surface of the shell and the negative sign is used for the inner surface.

A similar procedure is followed for the cylinder to which the ellipsoidal shell is attached. However, the resulting expressions are far more simple than those presented previously for the ellipsoidal shell, and the basic differential equations can be solved easily. To obtain the cylindrical shell equations, expressions (13) and (14) are substituted into Eqs (4) through (10). The equations (Ref 1) for the cylinder then appear as

$$
\begin{aligned}
& M_{x}=\frac{E t^{3}}{m^{4}} \frac{d W}{d x}, \\
& M_{y}=\mu M_{x}, \\
& N_{x}=0.25 \mathrm{PD},
\end{aligned}
$$

and

$$
\mathrm{N}_{\mathrm{y}}=0.5 \mathrm{D} \frac{\mathrm{Et}^{3}}{\mathrm{~m}^{4}} \frac{\mathrm{d}^{3} \mathrm{~W}}{\mathrm{dx}^{3}}+0.5 \mathrm{PD}
$$

where $t=$ thickness of the cylinder, and $x=$ axial coordinate of the cylinder.

For the cylinder, the Love-Meissner equations reduce to

$$
\frac{d^{4} w}{d x^{4}}+4 a^{4} w=0
$$

The solution of $\mathrm{Eq}(28)$ is given as

$$
w=e^{-a x}\left(C_{1} \sin a x+C_{2} \cos a x\right)+e^{a x}\left(C_{3} \sin a x+C_{4} \cos a x\right) \text {, }
$$

where $\mathrm{C}_{1}, \mathrm{C}_{2}, \mathrm{C}_{3}, \mathrm{C}_{4}$ are arbitrary constants, and $\mathrm{a}^{4}=\mathrm{m}^{4} / \mathrm{D}^{2} \mathrm{t}^{2}$.

Equations (22) and (23) are utilized to obtain the stresses in the cylinder after calculation of the moments and membrane forces from Eqs (24) through (27).

\section{SOLUTION OF THE ELLIPSOIDAL SHELL EQUATIONS}

Before proceeding to the.solution, it is convenient to change Eqs (19) and (20) slightly by multiplying each equation by $-\sin \theta / \mathrm{P}$ and by multiplying $\mathrm{Eq}(20)$ by $\mathrm{E}$ to give 


$$
M_{\theta}[\Gamma]-\mu(\sin \theta) \Gamma-g_{1}(\theta)(\sin \theta) \Omega=G(\theta)
$$

and

$$
M_{\theta}[\Omega]+\mu(\sin \theta) \Omega+g_{2}(\theta)(\sin \theta) \Gamma=0
$$

where

$$
\begin{aligned}
& M_{\theta}[Z]=-\frac{d}{d \theta}\left[(\sin \theta) v^{-2} \frac{d Z}{d \theta}\right]+\left(\cot ^{2} \theta\right)(\sin \theta) v^{2} Z, \\
& G(\theta)=-1 / 8 D_{\cdot}^{2} \beta^{2}\left(3 v^{2}+1\right)\left(1-v^{2}\right) \cos \theta, \\
& \Gamma=\Gamma(\theta)=v(\theta) / P, \\
& \Omega=\Omega(\theta)=E W(\theta) / P, \\
& g_{1}(\theta)=1 / 2 D T \beta v^{3}=s_{1}(\theta) / E,
\end{aligned}
$$

and

$$
g_{2}(\theta)=1 / 2\left[\mathrm{~m}^{4} \mathrm{D} \beta / \mathrm{T}^{3}\right] \mathrm{v}^{3}=s_{2}(0) \mathrm{D}
$$

The self-adjoint form of the operator $M_{\theta}$ has been used for general thin shells hy Pöschl (Ref 3):

From Eqs (29), (30), and (31), it is evident that there will be eight arbitrary constant to be determined in the solution of the problem of an ellipsoidal shell mounted on a long cylinder, since the solution of each. of the two parts involves four arbitrary constants. Thus, eight boundary conditions are needed, which are as follows: At the top of the ellipsoidal shell $\left(\right.$ at $\left.\theta=0^{\circ}\right)$ the shear and rotation are zero. Furthermore, the cylinder will be assumed to be semi-infinite in extent. Inspection of Eq (29) will show that, to insure finite results at the remote end of such a cylinder $(x \rightarrow \infty)$, the terms involving the positive exponential $e^{\text {ax }}$ must be dropped. This is done by setting $\mathrm{C}_{3}$ and $\mathrm{C}_{4}$ each equal to zero. The remaining four boundary conditions are obtained from the junction between the shell and the cylinder where continuity of axial moment, axial shear, deflection, and rotation must be maintained across the boundary.

Boundary Conditions

The boundary conditions can be summarized as follows:

For the Ellipse

$\Gamma(\theta), \Omega(\theta)=0$ at $\theta=0$

For the Cylinder

All quantities must be finite as $x \rightarrow \infty$

For the Junction

Equal Radial Moments:

$$
\left.\frac{2 \mathrm{~T}^{3} \beta^{2}}{\mathrm{D}} \frac{\mathrm{d} \Omega}{\mathrm{d} \theta}\right|_{n=i 1 / 2}=\alpha=\mathrm{t}^{3} \mathrm{a}\left(\mathrm{C}_{1}-\mathrm{C}_{2}\right)
$$

Equal Radial Deformation:

$$
T^{-1}\left[\left.2 \beta^{2} \frac{d \Gamma}{d \theta}\right|_{\theta=\pi / 2}+\frac{D^{2}}{4}\left(2-\beta^{2}-\mu\right)\right]=\gamma=t t^{-1}\left[\frac{D^{2} t^{3} a^{3}}{m^{4}}\left(C_{1}+C_{2}\right)+\frac{D^{2}}{4}(2-\mu)\right]
$$

Equal Axial Shear Forces:

$$
\left.\Gamma\right|_{\theta=\pi / 2}=-\frac{\ddot{D} a}{2 m}\left\{\alpha+\frac{m^{4}}{D^{2} a^{2}}\left[\gamma t-\frac{D^{2}(2-\mu)}{4}\right]\right\}
$$




$$
\left.\Omega\right|_{\theta=\pi / 2}=-\frac{1}{2 t^{3} a}\left\{\alpha-\frac{m^{4}}{D^{2} a^{2}}\left[\gamma t-\frac{D^{2}(2-\mu)}{4}\right]\right\}
$$

For the junction equations, $\alpha$ and $\gamma$ are defined by Eqs (34) and (35) and are considered unknowns until their values are determined toward the end of the problem. In Eqs (34) through (37) the left side of each equation represents the quantity for the ellipse at $\theta=\pi / 2$, and the right side of each equation represents the corresponding quantity for the cylinder at $x=0$.

As a result of the introduction of the quantities $\alpha$ and $\gamma$, the following expressions hold for the arbitrary constants in the cylinder equations:

$$
\begin{gathered}
C_{1}=\frac{1}{2 t^{3} a}\left\{\alpha+\frac{m^{4}}{D^{2} a^{2}}\left[\gamma t-\frac{D^{2}(2-\mu)}{4}\right]\right\} \\
C_{2}=\frac{-1}{2 t^{3} a}\left\{\alpha+\frac{m^{4}}{D^{2} a^{2}}\left[\gamma t-D^{2}(2-\mu)\right]\right\} \\
C_{3} C_{4}=0 \text { by condition (33). }
\end{gathered}
$$

\section{DIFFERENCE EQUATIONS}

An approximate solution for the ellipsoidal shell is obtained by using finite difference methods. The method of finite differences has been used in previous work in shell theory-notably by Au, Goodman, and Newmark (Ref.4). However, their work was primarily by hand computation; consequently, the methods of solution mentioned in this report (as well as the difference equations) are different.

Difference equations corresponding to the differential equations of the ellipsoidal shell [ Eqs (30) and (31)] are derived according to a method introduced by Varga (Ref 5). The boundary conditions [ Eq (32)] are needed for this derivation as well as two of the set of Eqs (34) through (3.7). The two chosen are Eqs (34) and (35). This introduces two unknowns, $\alpha$ and $\gamma$, which will be evaluated at a later time in the code with the use of the two remaining Eqs (36) and (37).

The interval $0 \leq \theta \leq \pi / 2$ is divided into $N$ subintervals (not necessarily equal) where

$$
h_{i}=\theta_{i}-\theta_{i-1}
$$

and

By way of notation, let

$$
0-\theta_{0}<\theta_{1}<\cdots<\theta_{N}-\pi / 2 \text {. }
$$

$$
\Gamma\left(\theta_{n}\right)=\Gamma_{n} ; \Omega\left(\theta_{n}\right)=\Omega_{n} \text {, etc. }
$$

Case I: $\theta_{\mathrm{n}}$ an Interior Point

This situation $\left(\theta_{n}\right.$ an interior point) occurs when $n \neq N$. (There is no equation corresponding to $\mathrm{n}=0$.) Then, at this point, the difference equation corresponding to the first differential $\mathrm{Eq}(30)$ is

$$
\begin{aligned}
& -\left(\frac{a_{n}+a_{n-1}}{h_{n}}\right) \Gamma_{n-1}+\left[\frac{a_{n}+a_{n-1}}{h_{n}}+\frac{a_{n+1}+a_{n}}{h_{n+1}}+\left(h_{n+1}+h_{n}\right) b_{n}\right] \Gamma_{n} \\
& -\left(\frac{a_{n+1}+a_{n}}{h_{n+1}}\right) \Gamma_{n+1}-\mu\left(\sin \theta_{n}\right)\left(h_{n+1}+h_{n}\right) \Gamma_{n} .
\end{aligned}
$$




$$
-g_{1}\left(\theta_{n}\right)\left(\sin \theta_{n}\right)\left(h_{n+1}+h_{n}\right) \Omega_{n}=\left(h_{n+1}+h_{n}\right) \vec{G}_{n} \text {. }
$$

where

and

$$
a(\theta)=(\sin \theta) v^{-2}
$$

(1)

$$
b(\theta)=\left(\cot ^{2} \theta\right)(\sin \theta) v^{2} ;
$$

thus

$$
a\left(\theta_{n}\right)=a_{n}
$$

and

$$
b\left(\theta_{n}\right)=b_{n} .
$$

The corresponding equation for $(31)$ is

$$
\begin{aligned}
& -\left(\frac{a_{n}+a_{n-1}}{h_{n}}\right) \Omega_{n-1}+\left[\frac{a_{n}+a_{n-1}}{h_{n}}+\frac{a_{n+1}+a_{n}}{h_{n+1}}+\left(h_{n+1}+h_{n}\right) b_{n}\right] \Omega_{n} \\
& -\left(\frac{a_{n+1}+a_{n}}{h_{n+1}}\right) \Omega_{n+1}+\mu\left(\sin \theta_{n}\right) \Omega_{n}+g_{2}\left(\theta_{n}\right)\left(\sin \theta_{n}\right)\left(h_{n+1}+h_{n}\right) \Gamma_{n}=0 .
\end{aligned}
$$

As a special case of the equations, if $n=1$, then the boundary conditions [Eq (32)], as well as the definition of $a(\theta)$, lead to

$$
\begin{aligned}
{\left[\frac{a_{1}}{h_{1}}+\frac{a_{2}+a_{1}}{h_{2}}+\left(h_{2}+h_{1}\right) b_{1}\right] } & \Gamma_{1}-\left(\frac{a_{2}+a_{1}}{h_{2}}\right) \Gamma_{2}-\mu\left(\sin \theta_{1}\right)\left(h_{2}+h_{1}\right) \Gamma_{1} \\
& -g_{1}\left(\theta_{1}\right)\left(\sin \theta_{1}\right)\left(h_{2}+h_{1}\right) \Omega_{1}=\left(h_{2}+h_{1}\right) G_{1},
\end{aligned}
$$

and a similar expression for $\delta(\theta), \Gamma(\theta)$ from $\mathrm{Eq}(39)$.

Case II: $\mathrm{n}=\mathrm{N}$

In this case, $\theta_{n}=\theta_{N}=\pi / 2$, and use is made of Eqs (34) and (35) to obtain

$$
\begin{aligned}
-\left(\frac{a_{N}+a_{N-1}}{i h_{N}}\right) \Gamma_{N-1}+\left(\frac{a_{N}+a_{N-1}}{h_{N}}+b_{N} h_{N}\right) \Gamma_{N}-\mu h_{N} \Gamma_{N} \\
-g_{1}\left(\theta_{P N}\right) h_{P N} \Omega_{N N}=h_{N N} G_{N N}+\frac{T a_{N}}{\beta^{2}} \gamma-\frac{D^{2} \cdot a_{N}}{4 \beta^{2}}\left(2-\beta^{2}-\mu\right)
\end{aligned}
$$

and

$$
\begin{array}{r}
-\left(\frac{a_{N}+a_{\bar{N}-1}}{h_{n}}\right) \Omega_{N-1}+\left(\frac{u_{\bar{N}} 1 a_{\bar{N}-1}}{h_{N}}+b_{N} h_{N}\right) \Omega_{N}+\mu h_{N} \Omega_{N} . \\
\quad+g_{2}\left(\theta_{N}\right) h_{N} \Gamma_{N}=a_{N}\left(T^{3} \beta^{L}\right)^{-1} D \alpha .
\end{array}
$$

Thus, the unknowns which occur in these equations are

$$
\left\{H_{i} \cdot\right\}_{i=1}^{N},\left\{\Omega_{i}\right\}_{i=1}^{N}, \cdot \alpha, \text { and } \gamma .
$$


The difference equations just derived can be written in matrix notation as follows:

$$
\begin{aligned}
& \text { A } \vec{\Gamma}-\mathrm{D}_{1} \vec{\Gamma}-\mathrm{D}_{2} \vec{\Omega}=\gamma \overrightarrow{\mathrm{P}}+\overrightarrow{\mathrm{Q}} \\
& \text { A } \vec{\Omega}+\mathrm{D}_{1} \vec{\Omega}+\mathrm{e}_{2} \vec{\Gamma}=\alpha \overrightarrow{\mathrm{R}}
\end{aligned}
$$

where

$A=N \times N$ matrix corresponding to the operator $M_{\theta}$ occurring in Eqs (30) and (31)

$\mathrm{D}_{1}=\mathrm{NxN}$ diagonal matrix with positive diagonal elements whose $\mathrm{n}^{\text {th }}$ element (along the major diagonal $n \neq N)$ is $\mu\left(\sin \theta_{n}\right)\left(h_{n+1}+h_{n}\right)$. The $N^{\text {th }}$ element is $\mu h_{N^{*}}$

$\mathrm{D}_{2}=\mathrm{NxN}$ diagonal matrix with positive diagonal elements whose $\mathrm{n}^{\text {th }}$ element (along the major diagonal $n \neq N)$ is $\left(\sin \theta_{n}\right) g_{l}\left(\theta_{n}\right)\left(h_{n+1}+h_{n}\right)$. The $N^{\text {th }}$ element is $g_{1}\left(\theta_{N}\right) h_{N^{*}}$

e $\quad=g_{2}(\theta) / g_{1}(\theta)$ is a positive constant.

$\vec{Q} \quad=$ Nxl matrix whose $n^{\text {th }}$ component $(n \neq N)$ is $G_{n}\left(h_{n+1}+h_{n}\right)$. The $N^{\text {th }}$ component is

$$
h_{N} G_{N}-\frac{D^{2} a_{N}}{4 \beta^{2}}\left(2-\beta^{2}-\mu\right)
$$

$\vec{P} \quad=$ Nxl matrix, all of whose components are zero except the $\mathrm{N}^{\text {th }}$ one. This one is

$$
\frac{\mathrm{T} \mathbf{a}_{\mathrm{N}}}{\beta^{2}}
$$

$\vec{R}=$ Nxl matrix, all of whose components are zero except the $N^{\text {th }}$ one. This one is

$$
a_{N}\left(T^{3} \beta^{2}\right)^{-1} D
$$

$\alpha, \gamma=$ previously defined scalars (unknown quantities at this point).

$\vec{\Gamma}, \vec{\Omega}=$ unknown Nxl vectors whose components are, respectively,

\section{GENERAL METHODS OF SOLUTION}

$$
\left\{\Gamma_{i}\right\}_{i=1}^{N} \text { and }\left\{\Omega_{i}\right\}_{i=1}^{N} \text {. }
$$

From the second equation of (43),

thus,

$$
\left[\ddot{\vec{\Omega}}=-o\left(A+D_{1}\right)^{-1} D_{2}\right] \vec{\Gamma}+\alpha\left(A+D_{1}\right)^{-1} \vec{R} ;
$$

which will be written as

$$
\left[A-D_{1}+e D_{2}\left(A+D_{1}\right)^{-1} D_{2}\right] \vec{\Gamma}=\gamma \vec{P}+\alpha D_{2}\left(A+D_{1}\right)^{-1} \vec{R}+\vec{Q},
$$

with

$$
T \vec{\Gamma}=\gamma \vec{P}+\alpha \vec{S}+\vec{Q}
$$

$$
T=\left[A-D_{1}+e D_{2}\left(A+D_{1}\right)^{-1} D_{2}\right] \text {, and } \vec{S}=D_{2}\left(A+D_{1}\right)^{-1} \vec{R} \text {. }
$$

The matrix Eq (45) may also be changed into another useful form by premultiplying by $\left(\dot{A}+D_{1}\right) D_{2}^{-1}$

Then, Eq (45) becomes

$$
T^{\prime} \vec{\Gamma}=\gamma \vec{P}^{\prime}+\alpha \vec{S}^{\prime}+\vec{Q}^{\prime}
$$


where

$$
T^{\prime}=\left[\left(A+D_{1}\right) D_{2}^{-1}\left(A-D_{1}\right)+e D_{2}\right]
$$

and

$$
\begin{aligned}
& \overrightarrow{P^{\prime}}=\left(A+D_{1}\right) D_{2}^{-1} \vec{P} \\
& \vec{S}^{\prime}=\left(A+D_{1}\right) D_{2}^{-1} \vec{S}=\vec{R} \\
& \vec{Q}^{\prime}=\left(A+D_{1}\right) D_{2}^{-1} \vec{Q} .
\end{aligned}
$$

There are two distinct SET codes, SET02 and SET03. Up to this point, no distinction has been made between these codes.

The difference in the two occurs in the methods used to solve the system (45) or (47) and is a result of the nature of the matrices $T$ and $T^{\prime}$.

Method (A): The SET02 Code

This method is based on Eq (47) with $T^{\prime}$ as defined in Eq (48). Three vectors must be found-

$$
\left(T^{\prime}\right)^{-1} \overrightarrow{P^{\prime}},\left(T^{\prime}\right)^{-1} \overrightarrow{S^{\prime}} \text {, and }\left(T^{\prime}\right)^{-1} \overrightarrow{Q^{\prime}}
$$

so that the system $T^{\prime} \vec{x}=\vec{k}$ must be solved for $\vec{x}$ for three different values of $\vec{k}$. Following a suggestion of $G$. Birkhoff, a direct method is used to solve the system $T^{\prime} \vec{x}=\vec{k}$ for $\vec{x}$, in contrast to an iterative method (about which more will be said later). A direct method seems particularly well suited to the matrix $T$ ' since "most" of the elements of $T$ ' are zero. A is tri-diagonal; thus, the same is true of $\left(A+D_{1}\right) D_{2}^{-1}$ and of $A-D_{1}$, so that the product $\left(A+D_{1}\right) D_{2}^{-1}\left(A-D_{1}\right)$ is a matrix whose only nonzero elements are on its main diagonal or on one of its nearest four parallel (to the main diagonal) diagonals. The same is consequently true also of

$$
T^{\prime}=\left[\left(A+D_{1}\right) D_{2}^{-1}\left(A-D_{1}\right)+e D_{2}\right] \text {. }
$$

Thus, $T^{\prime}=\left(t_{i, j}\right)$, with $t_{i, j}=0$ if $j \neq i-2, i-1, i, i+1,1+2$ and $i=3, \ldots, N-2$. A similar fact holds for $i=1,2, N-1$, and $N$.

The system $T^{\prime} \vec{x}=\vec{k}$ is now solved (for $\vec{x}$ ) by a method of elimination defined as follows:

1) The matrix $T^{\prime}$ is transformed into the matrix $U$ defined by $U=\left(u_{i, j}\right)$, where

$$
\begin{array}{cc}
u_{i, j}=t_{i, j} & i=1,2 \\
u_{i, j}=t_{i, j}-\frac{u_{i-1, j} t_{i, i-2}}{u_{i-1, i-2}}
\end{array}
$$

fur $i>3$ idud $j=1-1 ; 1,1+1,1+2$.

2) The $U$ is transformed into $U^{\prime}=\left(u_{i, j}^{\prime}\right)$ with

$$
\begin{aligned}
& u_{i, j}^{\prime}=u_{i, j} \quad \text { for } i=N-1, N \\
& u_{i, j}^{\prime}=u_{i, j}-\frac{u_{i+1, j}^{\prime} u_{i, i+2}}{u_{i+1, i+2}^{\prime}}
\end{aligned}
$$

for $i \leq N-2$ and $j=i-1, i, i+1$. 
3) The vector $\vec{k}$, with components $\left\{k_{i}\right\}_{i=1}^{N}$, is first transformed into the vector $\vec{l}$, with

$$
\begin{aligned}
\text { components }\left\{\ell_{i}\right\}_{i=1}^{N}, & \text { where } \\
\ell_{i} & =\left\{\begin{array}{l}
k_{i} i=1,2 \\
k_{i}-\frac{t_{i, i-2}}{u_{i-1, i-2}} \ell_{i-1}, i \geq 3,
\end{array}\right.
\end{aligned}
$$

and then $\vec{l}$ is transformed into the vector $\vec{m}$, with components $\left\{m_{i}\right\}_{i=1}^{N}$, with

$$
m_{i}=\left\{\begin{array}{l}
\ell_{i} \quad i=N-1, N \\
\ell_{i}-\frac{u_{i, i+2}}{u_{i+1}^{1}, i+2} m_{i+1}, i \leq N-2 .
\end{array}\right.
$$

The result of the se steps is the reduction of the original system $T^{\prime} \vec{x}=\vec{k}$ into the system

$$
U^{\prime} \vec{x}=\vec{m}
$$

where $U^{\prime}$ is now a tri-diagonal matrix. Thus, the system can now be solved for $\overrightarrow{\mathbf{x}}$ by the method which will be mentioned later in connection with the SET03 code.

Method (B): The SET03 Code

This method is based on Eq (45). It is clear that the vectors

$$
T^{-1} \vec{P}, T^{-1} \vec{S} \text {, and } T^{-1} \vec{Q}
$$

must be known to solve for $\vec{\Gamma}$. After these vectors are obtained, the relation

$$
\vec{\Gamma}=\gamma\left(T^{-1} \vec{P}\right)+\alpha\left(T^{-1} \vec{S}\right)+\left(T^{-1} \vec{Q}\right)
$$

yields each component of $\vec{\Gamma}$ as a simple function of $\alpha$, and $\gamma$. Equation (44) can be utilized to obtain $\vec{\Omega}$.

The process just described is based on the assumption that the two matrices, $A+D_{1}$ and $T$, can be inverted. By construction, $A$ is symmetric and positive definite, so that the addition of a diagonal matrix $D_{1}$ with positive diagonal elements results in a matrix $A+D_{1}$ which is also positive definite. In particular, $\left(A+D_{1}\right)^{-1}$ exists. Moreover, by construction, $A$ has nonzero elements only along its main diagonal and the two diagonals parallel to and nearest the main diagonal. $A$ is said to be tri-diagonal. The same is true of $\left(A+D_{1}\right)$. Such a matrix leads to a system of equations $\left(A+D_{1}\right) \vec{x}=\vec{k}$ which can be solved for $\vec{x}$ by a simple recursion relation (see, for example, Ref 6 , p 34). Thus, effectively, $\left(A+D_{1}\right)^{-1}$ of Eq (46) can be obtained in a fairly simple way.

On the other hand, $T$ is symmetric because, with $T$ as the transpose of $T$,

$$
T^{*}=\left[A-D_{1}+D_{2}\left(A+D_{1}\right)^{-1} D_{2}\right]^{*}=A-D_{1}+e D_{2}\left(A+D_{1}\right)^{-1} D_{2}=T
$$

since $A, D_{1}$, and $D_{2}$ are symmetric. 
Moreover,

$$
\begin{aligned}
T & =A+D_{1}+e D_{2}\left(A+D_{1}\right)^{-1} D_{2}-2 D_{1} \\
& =e^{1 / 2} D_{2}^{1 / 2}\left[e^{-1 / 2} D_{2}^{-1 / 2}\left(A+D_{1}\right) D_{2}^{-1 / 2}+e^{1 / 2} D_{2}^{1 / 2}\left(A+D_{1}\right)^{-1} D_{2}^{1 / 2}-2 e^{-1 / 2} D_{1} D_{2}^{-1}\right] D_{2}^{1 / 2} \\
& =e^{1 / 2} D_{2}^{1 / 2}\left[M+M^{-1}-2 e^{-1 / 2} D_{1} D_{2}^{-1}\right] D_{2}^{1 / 2},
\end{aligned}
$$

where

$$
M=e^{-1 / 2} D_{2}^{-1 / 2}\left(A+D_{1}\right) D_{2}^{-1 / 2}
$$

is symmetric and positive definite. Now if $K=M+M^{-1}$, then $K$ is symmetric and positive definite and its eigenvalues are of the form $\lambda+\lambda^{-1}$, where $\lambda$ is an eigenvalue of $M$. Thus, since $\lambda>0$, the minimum of $\lambda+\lambda^{-1}$ is 2 and, therefore, a lower bound for the eigenvalues of $K$ is 2 . Now,

where

$$
(T \vec{x}, \vec{x})=e^{1 / 2}\left\{(K \vec{y}, \vec{y})-2 e^{-1 / 2}\left(D_{1} D_{2}^{-1} \vec{y}, \vec{y}\right)\right\} \text {. }
$$

$$
\vec{y}=D_{2}^{1 / 2 \vec{x}}
$$

Again,

$$
(K \vec{y}, \vec{y}) \geq 2(\vec{y}, \vec{y})
$$

and

$$
e^{-1 / 2}\left(D_{1} D_{2}^{-1} \vec{y}, \vec{y}\right) \leq \zeta(\vec{y}, \vec{y})
$$

where $\zeta$ is the largest eigenvalue of $e^{-1 / 2} D_{1} D_{2}^{-1}$. However, $e^{-1 / 2} D_{1} D_{2}^{-1}(a$ diagonal matrix) has elements $2 \mu / \mathrm{m}^{2}\left(\frac{\mathrm{D}}{\mathrm{T}}\right) \mathrm{v}_{\mathrm{n}}^{3} \beta$. The minium of $\mathrm{v}^{3}$ is $1 / \beta^{3}$, so that (assuming $\beta \geq 1$ ):

$$
\zeta \leq \frac{2 \mu \beta^{2}}{m^{2}\left(\frac{D}{T}\right)} .
$$

Thus,

$$
(T \vec{x}, \vec{x}) \geq e^{1 / 2}\left[2-\frac{4 \mu \beta^{2}}{m^{2}\left(\frac{D}{T}\right)}\right]:(\vec{y}, \vec{y}) .
$$

Now,

$$
\mathrm{e}^{1 / 2}=\frac{\mathrm{m}^{2}}{\mathrm{~T}^{2}}
$$

and

$$
(\vec{y} ; \vec{y})=\left(D_{?} \vec{x} ; \vec{x}\right) \geq \eta(\vec{x}, \vec{x})
$$

where $\eta$ is a lower bound for the eigenvalues of $\mathrm{D}_{2} \cdot$ Now $\mathrm{D}_{2}$ is a diagonal matrix with elements

$$
\frac{1}{2}\left(\sin \theta_{n}\right) \operatorname{DT} \beta v_{n}^{3}\left(h_{n+1}-h_{n}\right)
$$

except for the $\mathrm{N}^{\text {th }}$ element which is $\frac{1}{2} \mathrm{DT} \beta \mathrm{v}_{\mathrm{N}}^{3} \mathrm{~h}_{\mathrm{N}}$. Since

$$
\sin \theta_{1} \simeq \theta_{1}=\theta_{1}-\theta_{0}=h_{1} \text {, }
$$

it can be assumed, in general*, that $\eta$ can be chosen as DT $\beta h_{l}^{2}$.

\#This is not precisely so in all cases. We will, however, make this assumption. 
'Thus,

$$
(T \vec{x}, \vec{x}) \geq 2 \beta h_{1}^{2}\left[m^{2}\left(\frac{D}{T}\right)-2 \mu \beta^{2}\right](x, x) .
$$

Clearly then, $T$ is positive definite when

or

$$
\Lambda=m^{2}\left(\frac{D}{T}\right)-2 \mu \beta^{2}>0
$$

$$
1 \leq \beta^{2}<\sqrt{3}\left(\frac{1-\mu^{2}}{\mu^{2}}\right)^{1 / 2}\left(\frac{\mathrm{D}}{\mathrm{T}}\right)
$$

This is not a severe restriction. For example, for $\mu=0.3$, this states that

$$
1 \leq \beta^{2}<\sqrt{30}\left(\frac{D}{T}\right)
$$

and $\frac{D}{T}$ is usually $\geq 10$. Moreover, if a is the smallest eigenvalue of $T$, then it can be said that, from Eq (5l),

$$
a \geq 2 \beta h_{1}^{2} \Lambda
$$

It is not clear what happens for values of $\beta$ other than those satisfying. Eq (51). Although the code SET03 is not internally restricted to these values of $\beta$, it will nevertheless be assumed for the following theory that $\beta$ does satisfy Eq (51).

The method used in SET03 to solve the system $T \vec{x}=\vec{k}$ is an iterative method in contrast to the direct method used in SET02. The primary advantage of this iterative method is its inherent stability with respect to round-off errors. Its primary disadvantage (a major one) is the time neces sary to complete a problem in contrast to a direct method. This will be discussed in detail later.

The method to be used depends strongly on the fact that $\mathrm{T}$ is positive definite. Let

and

$$
\mathrm{b}=\text { largest eigenvalue of } \mathrm{T}
$$

Then, the new matrix

$$
a=\text { smallest eigenvalue of } \mathrm{T} \text { (as before). }
$$

$$
\left[\mathrm{I}-\frac{2}{\mathrm{a}+\mathrm{b}} \mathrm{T}\right]
$$

is formed which now has eigenvalues in the region $-1<\frac{a-b}{a+b} \leq \lambda \leq \frac{b-a}{a+b}<1$. With this new matrix, polynomial operators are used with Chebyshev polynomials in the manner introduced by Shortley (Ref 7), which leads to the following iterative scheme. Let

$$
\begin{aligned}
& \vec{u}_{0}=\vec{x}_{0}=\text { initial guess, } \\
& \vec{u}_{1}=\left[I-\frac{2}{a+b} T\right] \vec{u}_{0}+\frac{2}{a+b} \vec{k},
\end{aligned}
$$

and

$$
T_{n}(d) \vec{u}_{n} \equiv 2 d T_{n-1}(d)\left[I-\frac{2}{a+b} T\right] \vec{u}_{n-1}-T_{n-2}(d) \vec{u}_{n-2}+\frac{4 d T_{n-1}(d)}{a+b} \vec{k}
$$

for $n=2,3, \ldots$. Now,

$$
\mathrm{d}=\frac{\mathrm{a}+\mathrm{b}}{\mathrm{b}-\mathrm{a}}>1
$$

and

$$
T_{0}(d)=1, \quad T_{1}(d)=d
$$


with

$$
T_{n}(d)=2 d T_{n-1}(d)-T_{n-2}(d)
$$

for $n=2,3, \ldots$. Let $\|\vec{x}\|=\sqrt{\sum_{i} x_{i}^{2}}$ where $\left\{x_{i}\right\}$ is the sét of components of the vector $\vec{x}$. Also let .

$$
\vec{\epsilon}_{n}=\vec{x}_{1}-\vec{u}_{n}
$$

Then it can be shown that *

$$
\left\|\vec{\epsilon}_{n}\right\| \leq\left[T_{n}(d)\right]^{-1}\left\|\vec{\epsilon}_{o}\right\|
$$

so that if $T_{N}(d) \geq 50$, then $\vec{u}_{N}$ will be less than $1 \%$ from the answer, provided the initial vector $\vec{u}_{0}=\vec{x}_{0}^{0}$ is within $50 \%$ of the answer.

This code makes use of the result of SET02 as the initial vector $\vec{u}_{0}$. An estimate of the largest eigenvalue of $T$ is obtained from the recursion relation

$$
\begin{aligned}
T \vec{x}_{n-1} & =\vec{S}_{n} \\
\lambda_{n} & =\left(\vec{S}_{n}, \vec{x}_{n-1}\right) /\left(\vec{x}_{n-1}, \vec{x}_{n-1}\right) \\
\vec{x}_{n} & =\vec{S}_{n} / \lambda_{n}
\end{aligned}
$$

for $n=1,2,3, \ldots M$. (M is set at 50.) The symbol $\overrightarrow{(x}, \vec{y})$ indicates the inner product of $\vec{x}$ and $\vec{y}$ and is defined as

$$
\overrightarrow{(x}, \vec{y})=\sum_{i} x_{i} y_{i}
$$

where $\left\{x_{i}\right\},\left\{y_{i}\right\}$ are, respectively, the sets of components of $\vec{x}$ and $\vec{y}$. The number used for $b, b_{1}$. is then set at $(1 \cdot 1) \lambda_{M^{*}}$ It is important that this number be greater than or equal to $b$. An estimate, $a_{1}$, of the number a (smallest eigenvalue of ' $T$ ) is obtained from the Eqs (53) by using the matrix $T-b_{1} I$ instead of $T$. The number obtained at the end of $M$ iterations, call it $\lambda_{M}^{\prime}$, gives rise to the estirinate

$$
a_{1}=\lambda_{M}^{\prime}+b_{1}
$$

This value is used unless it is smaller than the estimate of Eq (52), in which case this latter value is used. Unlike the estimate for the largest eigenvalue, which had to be larger than the actual value, no such restriction exists for the estimate $a_{1}$.

\section{CALCULATION OF THE STRESSES}

From Eqs (15) through (18), the equations for the resultant stresses and stress couples for the ellipsoidal shell can be found in terms of $\Gamma(\theta)$ and $\Omega(\theta)$ in the following equations. It should be mentioned that only normalized resultant stresses and stress couples are obtained, namely $N_{x} / P$, $N_{y} / P, M_{x} / P$, and $M_{y} / P$. This will lead to the normalized stresses $\sigma_{x} / P$ and $\sigma_{y} / P$.

$$
\begin{gathered}
\left(\frac{N_{x}}{P}\right)_{n}=\frac{2}{D \delta_{n}}\left(\cot \theta_{n}\right) \Gamma_{n}+{ }_{4}^{1} D \delta_{n} \\
\left(\frac{N}{P}\right)_{n}=\frac{2}{D \delta_{n}^{1}}\left(\frac{d \Gamma}{d \theta}\right)_{n}+\frac{1}{4} D\left[\frac{2 \delta_{n}^{2}-\beta^{2}}{\delta_{n}}\right]
\end{gathered}
$$

* See, for example, a similar analys is by D. Young in Ref 8. 


$$
\begin{aligned}
& \left(\frac{M_{x}}{P}\right)_{n}=\frac{2 T^{3}}{m^{4} D \delta_{n}}\left[\frac{1}{v_{n}^{2}}\left(\frac{d \Omega}{d \theta}\right)_{n}+\mu\left(\cot \theta_{n}\right) \Omega_{n}\right] \\
& \left(\frac{M y}{P}\right)_{n}=\frac{2 T^{3}}{m^{4} D \delta_{n}}\left[\frac{\mu}{v_{n}^{2}}\left(\frac{d \Omega}{d \theta}\right)_{n}+\Omega_{n}\left(\cot \theta_{n}\right)\right]
\end{aligned}
$$

where the subscript $\mathrm{n}$ indicates, as before, the evaluation of the function at $\theta=\theta_{n}$.

The functions $\left(\frac{d \Omega}{d \theta}\right)_{n}$ and $\left(\frac{d \Gamma}{d \theta}\right)_{n}$ are evaluated from the following formulas:

For $n \neq N$,

$$
\left(\frac{d \Omega}{d \theta}\right)_{n}=\frac{h_{n}}{h_{n+1}^{2}+h_{n} h_{n+1}} \Omega_{n+1}+\frac{h_{n+1}-h_{n}}{h_{n+1} h_{n}} \Omega_{n}-\frac{h_{n+1}}{h_{n}^{2}+h_{n} h_{n+1}} \Omega_{n-1} .
$$

For $\mathrm{n}=\mathrm{N}$,

$$
\left(\frac{d \Omega}{d \theta}\right)_{N}=\frac{{ }^{2 h_{N}}+h_{N-1}}{h_{N}^{2}+h_{N} h_{N-1}} \Omega_{N}-\frac{h_{N}+h_{N-1}}{h_{N} h_{N-1}} \Omega_{N-1}+\frac{h_{N}}{h_{N-1}^{2}+h_{N} h_{N-1}} \Omega_{N-2} .
$$

Similar expressions are used for $\left(\frac{d \Gamma}{d \theta}\right)_{n}$.

The stresses are now obtained by using the formulas of Eqs (22) and (23):

For the cylinder, the following relations hold:

$$
\begin{gathered}
\frac{M_{x}}{P}=\frac{a t^{3}}{m^{4}} e^{-a x}\left[C_{1}(\cos a x-\sin a x)-C_{2}(\cos a x+\sin a x)\right], \\
\frac{M_{y}}{P}=\mu \frac{M_{x}}{P}, \\
\frac{N_{y}}{P}=\frac{D t^{3} a^{3}}{m^{4}} e^{-a x}\left[C_{1}(\cos a x+\sin a x)+C_{2}(\cos a x-\sin a x)\right]+\frac{D}{2},
\end{gathered}
$$

and

$$
\frac{N_{x}}{P}=\frac{D}{4}
$$

where

and

$$
C_{1}=\frac{1}{2 t^{3} a}\left\{\alpha+\frac{m^{4}}{D^{2} a^{2}}\left[\gamma t-\frac{D^{2}(2-\mu)}{4}\right]\right\}
$$

$$
C_{2}=\frac{1}{2 t^{3} a}\left\{\alpha-\frac{m^{4}}{D^{2} a^{2}}\left[\gamma t-\frac{D^{2}(2-\mu)}{4}\right]\right\}
$$

The stresses in the cylinder can then be obtained from the formulas of Eqs (22) and (23), with $\mathrm{T}$ now replaced by $\mathrm{t}$.

The cylinder has been assumed infinitely long in the previous equations. Since the discontinuity stresses caused by the juncture reach.steady values in one period of the trigonometric functions shown previously, the stresses will only be calculated for a distance from the juncture corresponding to one period. This distance is calculated from a $L=2 \pi$. Since a has been shown to depend on the 
dimensions and material of the cylinder, the length $L$ is different for each problem. $L$ will be determined for each problem, and the axial and radial stresses on the inner and outer surfaces of the cylinder will be calculated for any specified number of points, not necessarily equally spaced along its length.

\section{INPUT PREPARATION}

The input for the two codes is the same except for the code designation. A sample input is shown in the Appendix. The input is as follows:

TITLE Card: Columns 1-67 are available for problem identification. Columns 68-72 must contain either SET02 or SET03, where the next to last character is a zero.

Card 1001: This card contains Poissons' ratio.

Card 2001: This card is used to specify the following:

1) The number of intervals, an integer, into which the ellipse is divided by the mesh: $5 \leq \mathrm{n} \leq 500$.

2) $\beta$, the ratio of the major diameter to the minor diameter for the ellipse.

3) $D$, the cylinder diameter.

4) $\mathrm{T}$, the ellipse thickness.

Card 3001: This card is used to define the mesh in the ellipse by pairs of numbers. The first number of each pair, an integer, indicates the number of intervals in a given region. The second number of each pair indicates the angle, in degrees, at which the region terminates. Each region is assumed to start at the angle where the last region terminated; the first region is assumed to start at zero. The angle used for input is that formed by the major axis of the ellipse and a line through the center of the ellipse intersecting the shell. Thus, this angle is not the angle $\theta$ used in the difference equations. In fact, if $\varphi$ is the angle used in the input, then $\varphi$ and $\theta$ are connected by the relation

$$
\varphi=\arctan \left[\frac{1}{\beta^{2}} \cot \theta\right] .
$$

Thus, for example, $\varphi=0$ and $\theta=\pi / 2$ are corresponding angles. 'l'here can be a maximum of ten regions in the ellipse, and the last one must terminate with an angle of 90 degrees. The sum of the intervals on this card must equal the number of intervals specified on card 2001.

Card 4001: This card contains two quantities: the number of intervals (an integer) to be used in the cylinder in the first period of behavior, and the cylinder thickness.

Card 5001: This card is used to define the mesh in the cylinder by pairs of numbers in a manner similar to that used in the ellipse. The first number of each pair, an integer, gives the number of intervals in a region. The second number of each pair indicates the fraction of the first period of behavior to which the region extends. As in the ellipse, each region is assumed to start where the previous region terminated, the first region starting at zero. There can be a maximum of ten regions, the last of which must have a termination fraction of 1.0. The sum of the intervals must equal the number of intervals specified on card 4001 .

All of the card numbers, as well as those quantities designated as integers, must not contain a decimal point. All other input quantities must have a decimal point some where in the number. For example, the value .00125 can be written as $.00125, .125 E-2$, or $1.25 E-3$. 
Values on each card, other than the title card, must start in column.l and must be separated by commas. The last number on a card must not be followed by a comma. The first blank column on any card other than the title card indicates the end of data on that card.

\section{OUTPUT DESCRIPTION}

A sample output is shown in Appendix I. The output tape must be printed on the 717 tape-toprinter under program control. Each page of output contains the problem identification, the code designation, and the page number.

On page one, all of the input data except mesh description is printed along with $\alpha$ and $\gamma$. Page one will also contain the estimate for the upper bound of the eigenvalues and the number of terms of the recursion formula needed for convergence for each of the three vectors.

On page two, the normalized stress values for the ellipse begin. The axial and circumferential components on the outer and inner surfaces are tabulated as functions of angle, as specified by the input, from the top of the ellipse down to the juncture.

The cylinder stresses are tabulated in much the same manner except that they are a function of distance from the juncture rather than angle.

\section{CONCLUSIONS}

The two codes have certain characteristics which are very useful in obtaining results which can be regarded as reasonably accurate. In the first place, a typical problem is run on the SET02 code much faster than on the SET03 code. On the other hand, the SET02 code is subject to roundoff errors when the mesh is sufficiently refined, while the method used in the SET03 code is inherently "stable" in the sense that an error introduced at the $\mathrm{m}^{\text {th }}$ step $\mathrm{w}$ ill decrease to zero as the number of iterations is increased. This fact is particularly useful when a mesh is being determined for a certain problem or a class of problems to be run on SET02. On the one hand, the mesh should be fine enough to insure a reasonable approximation to the solution of the differential equation; on the other hand, it should not introduce so many points that round-off will play a significant role in the results.

Care should be exercised in choosing a mesh because of the transformation of the independent variable in the code-namely, the change from $\varphi$ to $\theta$. If $h_{i}^{\prime}$ is the mesh in the angle $\varphi$ and $h_{i}$ is the mesh in the angle $\theta$, then,

where

$$
h_{i}^{\prime}\left[\frac{\beta^{2}}{1+\left(\beta^{4}-1\right) \sin ^{2} \varphi}\right]=h_{i}
$$

$$
\varphi_{i} \leq \varphi \leq \varphi_{i-1}
$$

with

$$
\theta_{i}-\theta_{i-1}=\hat{h}_{i}, \varphi_{i-1}-\varphi_{i}=\dot{h}_{i}^{\prime} \text {, and } \varphi_{i}=\arctan \left[\frac{1}{\beta^{2}} \cot \theta_{i}\right] \text {. }
$$

Thus, at the top, $\varphi=90^{\circ}$ and $0=0^{\circ}$ so that

$$
h_{i} \simeq \frac{1}{\beta^{2}} h_{i}^{\prime}
$$

and at the juncture, $\theta=90^{\circ}$ and $\varphi=0^{\circ}$,

$$
h_{i} \simeq \beta^{2} h_{i}^{\prime} ;
$$

all of which means that the mesh the code is using may be very much finer or coarser than the user might have intended. It has been the experience of the authors that the mesh in the neighborhood of 
the juncture should be finer than that at the top of the ellipsoid. The two codes have also been used to check each other's results for given problems. This was necessary inasmuch as analytic results were not available for any but hemispherical heads. Hemispherical heads correspond to $\beta=1$, and some results for this type of shell are available from Watts and Lang (Ref 9). To illustrate the type of accuracy obtainable in the code SET02, and also to check the code itself, some problems from

Ref 9 were run, and the comparison is presented in Table I.

The mesh used consists of 54 points distributed as follows (measured in the angle $\varphi$ ):
$1 / 2$ degree intervals to 10 degrees
1 degree intervals to 16 degrees
2 degree intervals to 24 degrees
4 degree intervals to 72 degrees
2 degree intervals to 84 degrees
1 degree intervals to 90 degrees

The stresses given are for both the hemisphere and the cylinder at the juncture $\left(\varphi=0^{\circ}\right)$ and are normalized, as is done by Watts and Lang, by dividing through by $\mathrm{D} / 2 \mathrm{t}$. By way of notation, a subscript $o$ or $i$ is used in the stresses to indicate the outside or the inside surfaces, respectively.

\section{TABLE I - COMPARISON OF PROBLEM RESULTS}

Hemisphere

\begin{tabular}{|c|c|c|c|c|c|c|}
\hline & \multirow[b]{2}{*}{$\frac{2 \mathrm{t} \sigma_{\mathrm{xO}}}{\mathrm{DP}}$} & \multirow[b]{2}{*}{$\frac{2 t \sigma_{x i}}{D P}$} & \multirow[b]{2}{*}{$\frac{2 \mathrm{t} \sigma}{\mathrm{yP}}$} & \multirow[b]{2}{*}{$\frac{2 t \sigma_{y i}}{D P}$} \\
\hline D & $\mathrm{T}$ & $\mathbf{t}$ & & & & \\
\hline 32 & .8 & 1.0 & .6767 & .5733 & .8583 & .8273 \\
\hline 16. & .8 & 1.0 & .6772 & .5728 & .8582 & .8268 \\
\hline 20 & 1.0 & 1.0 & .5007 & 49.93 & .7496 & .7492 \\
\hline 16 & 1.6 & 1.0 & .2359 & .3891 & .5619 & .6079 \\
\hline \multicolumn{3}{|c|}{ Cylinder } & & . & & \\
\hline 32 & .8 & 1.0 & .5331 & .4669 & .8152 & .7954 \\
\hline 16 & .8 & 1.0 & .5334 & .4666 & .8150 & .7950 \\
\hline 20 & 1.0 & 1.0 & .6007 & .1993 & .7196 & .7102 \\
\hline 16 & 1.6 & 1.0 & $.30 \cdot 10$ & $\therefore 8560$ & .5024 & . ซல்ப் \\
\hline
\end{tabular}

\begin{tabular}{|c|c|c|c|}
\hline $\begin{array}{c}2 \mathrm{t} \sigma_{\mathrm{xO}} \\
\mathrm{DP}\end{array}$ & $\begin{array}{c}2 \mathrm{t} \sigma_{\mathrm{xi}} \\
\mathrm{DP}\end{array}$ & $\frac{2 t \sigma_{\text {yo }}}{\mathrm{DP}}$ & $\frac{2 t \sigma_{y i}}{\mathrm{DP}}$ \\
\hline .6737 & .5763 & .8585 & .8293 \\
\hline .6822 & .5678 & .8569 & $.8226^{\circ}$ \\
\hline .5048 & .4952 & .7477 & .7449 \\
\hline .2388 & .3862 & .5587 & .6029 \\
\hline .5318 & .4682 & .8159 & .7968 \\
\hline .5369 & .4631 & .8133 & . 7911 \\
\hline .5053 & .4747 & .7479 & .7447 \\
\hline . JILI & .6874 & .5807 & .6934 \\
\hline
\end{tabular}

\section{OPERATING.INSTRUCTIONS}

Either program may be operated from cards, a program tape, or a service tape.

1) Ready program in one of the given forms.

2) Ready a tape on logical 5 for output.

3) Ready all problems in reader (a blank card must follow each problem).

4) All sense switches on console must be up.

5) Press CLEAR on the console and load the program.

There is only one stop in the code at 110 octal; the on-line comment will instruct the operator.

The output tape which must be printed on program control is not rewound nor is an end-of-file written on it at any time by the program.

\section{Code Restrictions}

1) Number of intervals in ellipse: $5 \leq \dot{n} \leq 500$

2) Number of regions in ellipse $\leq 10$

3) Number of regions in cylinder $\leq 10$ 
Machine Requirements

Core Size

Tapes

Drums

Punch

On-Line Printer

Off-Line Printer
32768 words

2

none

none

with SHARE 2 or GL OUT2 Board

yes 


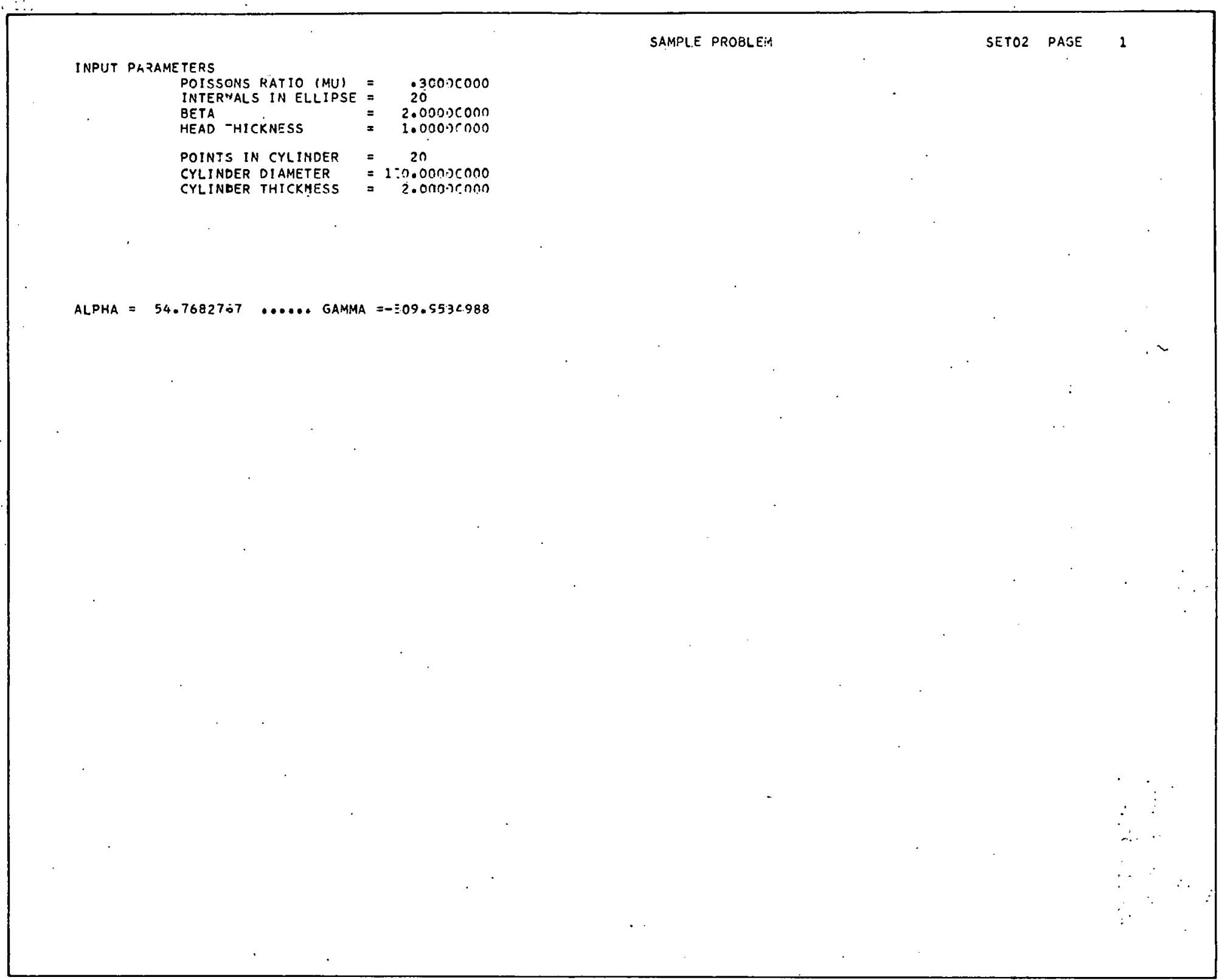




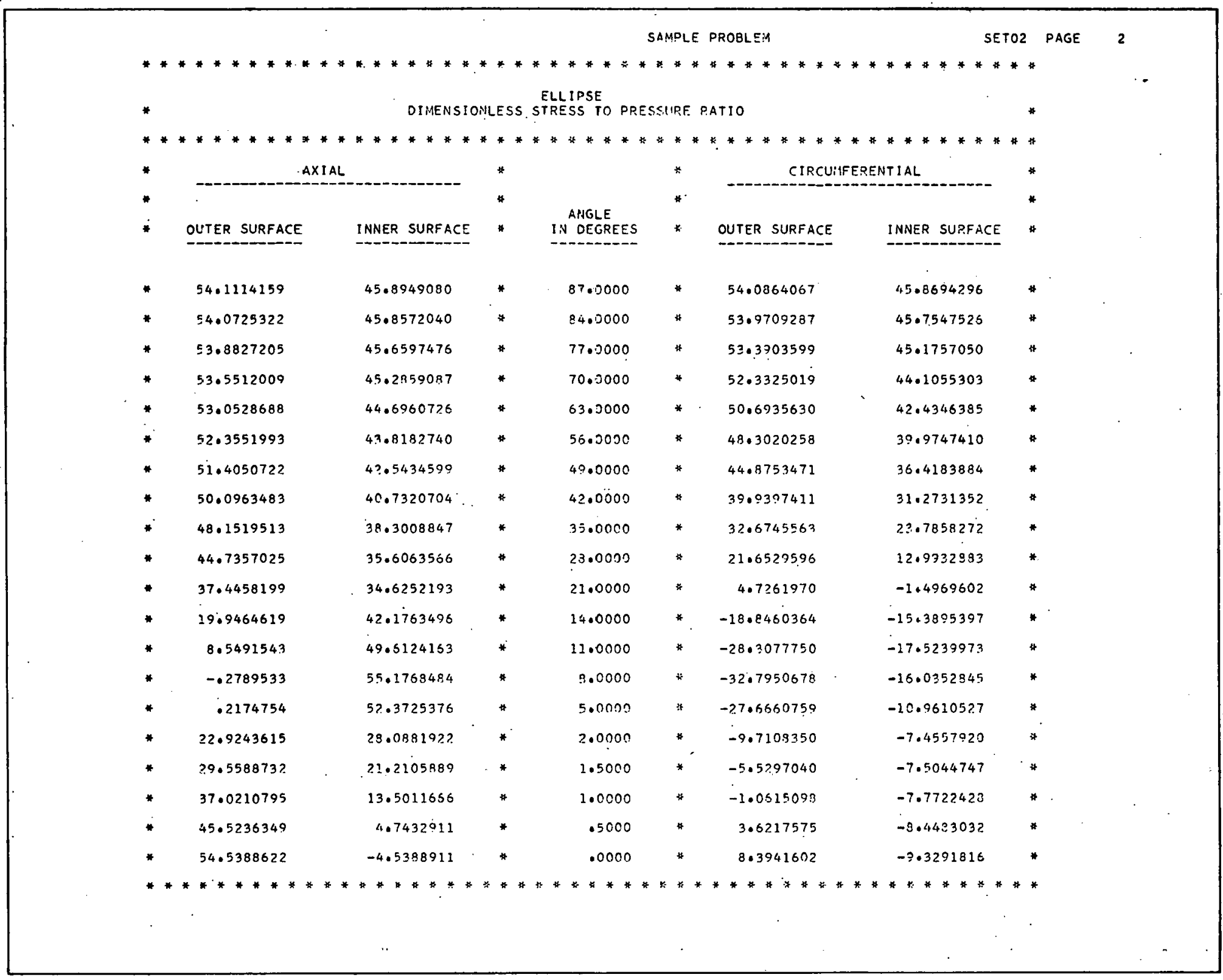




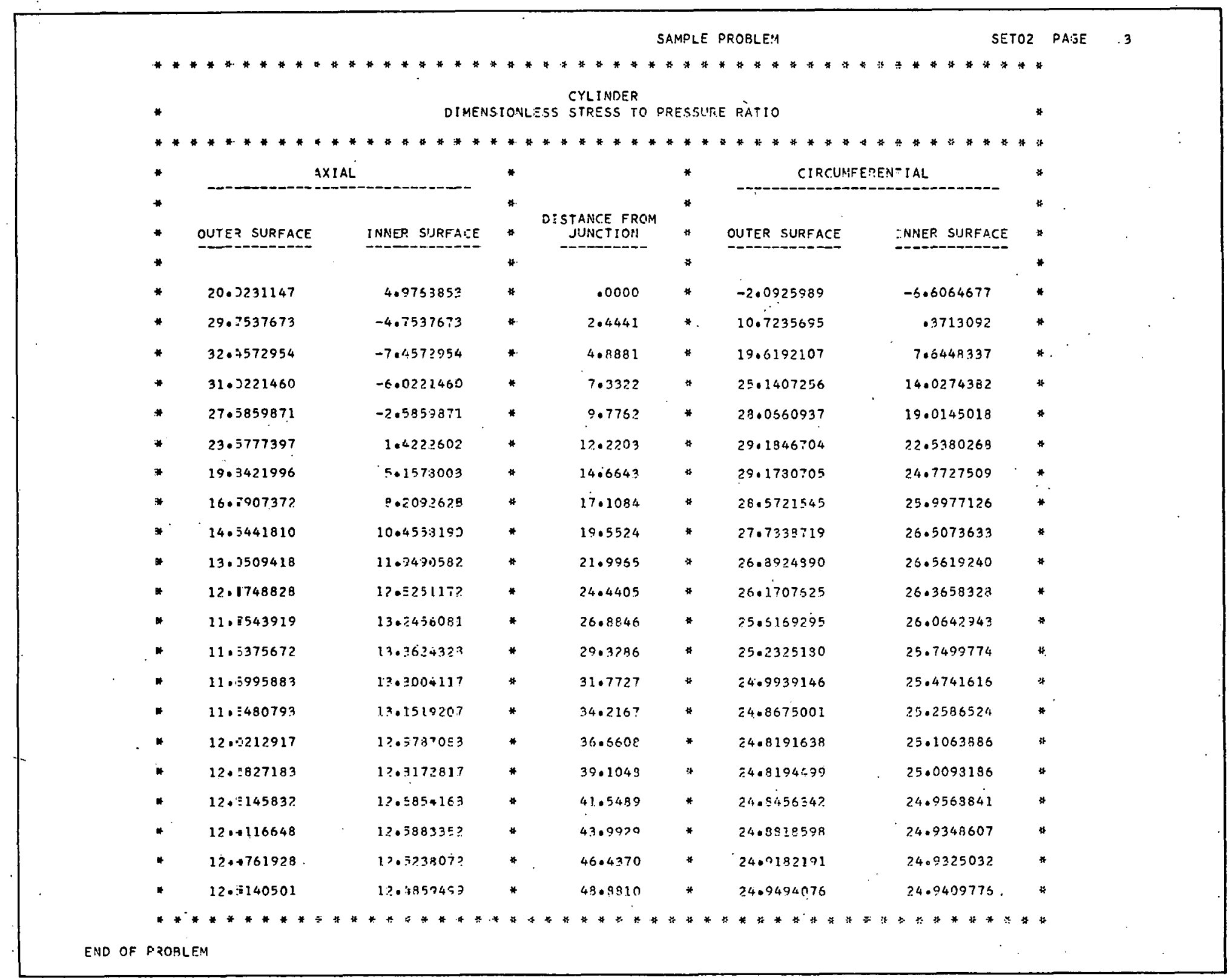




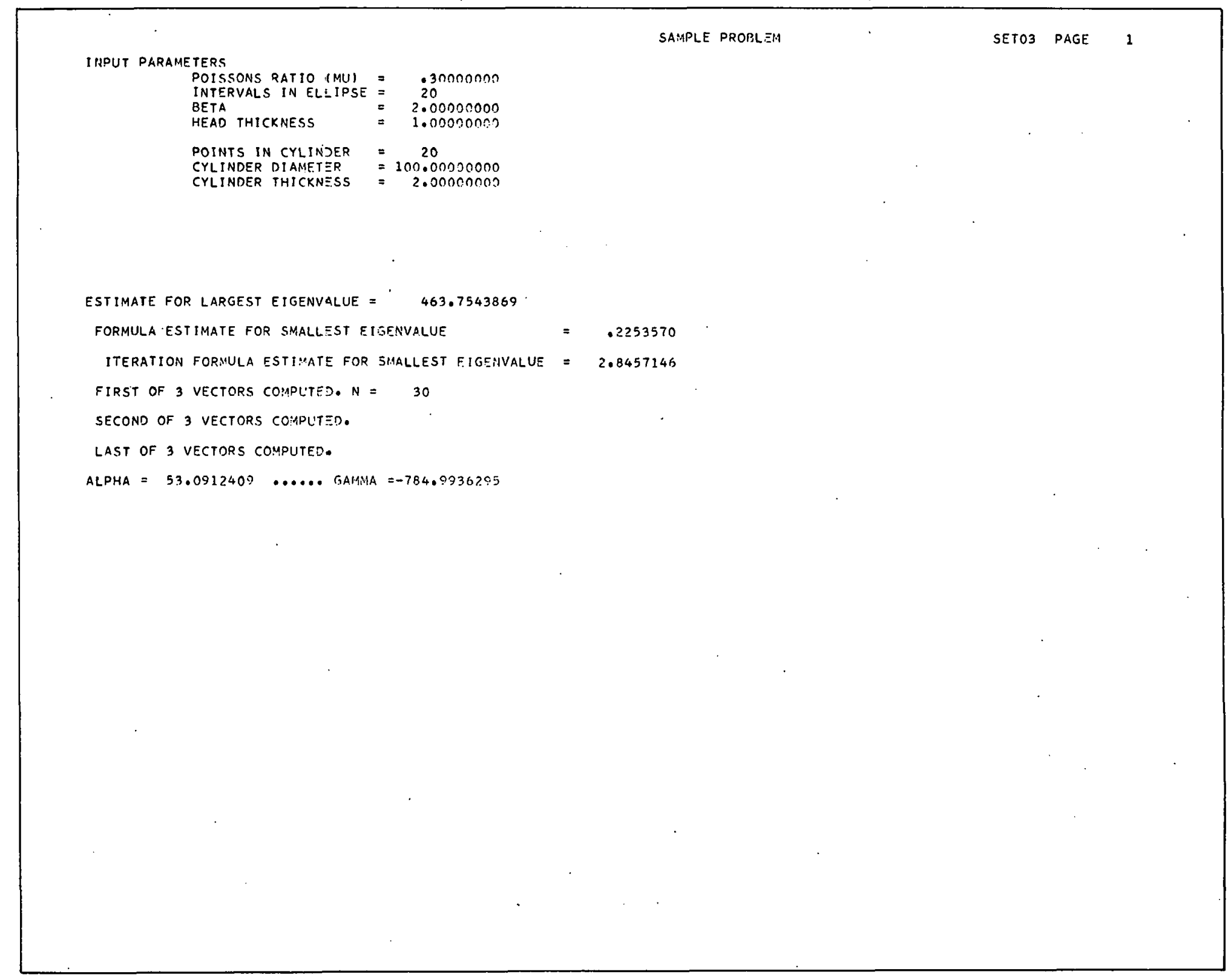


DIMEASIONLESS STRESS TO PRESSIJRE RETIO

- Axial

* outer surface

54.2201042

- $\quad 54.1811323$

* $\quad 53.9910164$

- $\$ 3.6591511$

* 53.1603737

* $\quad 52.4622550$

* $\quad 51.5119863$

$\quad 5.0 .2042837$

* 48.2642779

* 44.8599262

- 37.5963072

- 20.0728560

8.6059723

- $\quad-.3715415$

* $\quad-.1345537$

* $\quad 22.2283425$

- $\quad 28.9032207$

- $\quad 36.2067304$

* 44.6533904

- 53.5153455

$-3.615573 \varepsilon$

$45.785-595$

$45.7<8: 375$

45.5509834

45.1775112

$44.588:-891$

43.7100914

$42.43600 \varepsilon \varepsilon$

$40.624-145$

38.1900258

$35.484042 C$

$34.482769 \mathrm{C}$

42.065 .768

$49.5050 \times 423$

$55.255 \div 611$

$52.730 .26 ?$

28.7774666

21.9615428

14.3125991

5.6126718
CIRCUMFERENTIAL

\section{ANGLE}

IN DEGREES

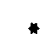

INNER SLARFACE

$\begin{array}{rrr}87.0000 & * & 54.1951127 \\ 84.0000 & * & 54.0795808 \\ 77.0000 & * & 53.4988065 \\ 70.0000 & * & 52.4406872 \\ 63.0000 & * & 50.8014379 \\ 56.0000 & * & 48.4096127 \\ 49.0000 & * & 44.9828472 \\ 42.0000 & * & 40.0477800 \\ 35.0000 & * & 32.7842464 \\ 28.0000 & * & 21.7644043 \\ 21.0000 & * & 4.8307996 \\ 14.0000 & * & -19.8026934 \\ 11.0000 & * & -28.3310194 \\ 8.0000 & * & -32.9003062 \\ 5.0000 & * & -27.8446231 \\ 2.0000 & * & -9.8747373 \\ 1.5000 & * & -5.6597529 \\ 1.0000 & * & -1.1678276 \\ .5000 & * & 3.5490099 \\ .0000 & * & 8.3948201\end{array}$

INNER SURFACE

OUTER SURFACE

45.7602572

45.6456295

45.0668159

43.9970331

42.3267837

39.8678570

36.3128738

31.1633649

23.6833189

12.8877215

$-105191917$

$-15.5531468$

$-17.6991502$

$-16.1763732$

$-10.98 ? 907 ?$

$-7.2179494$

$-7.1919335$

$-7.3961839$

$-8.0015255$

$-8.7744111$ 


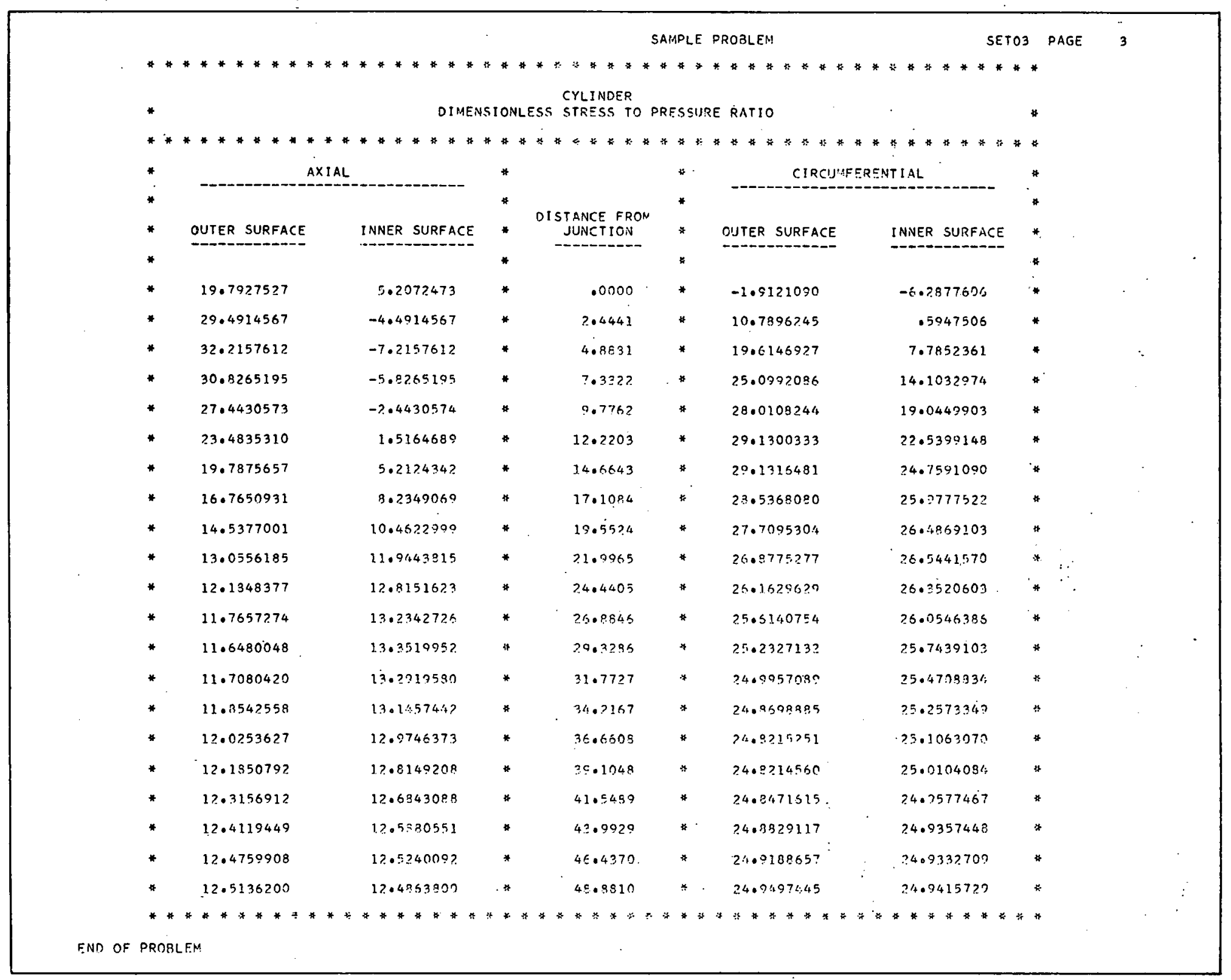




\section{ACKNOWLEDGMENT}

The authors are indebted to Mr. W. R. Burrows of the Standard Oil Company of Indiana and to Dr: G. D. Galletly of the Shell Development Company for their help during the course of this work.

\section{REFERENCES}

1. G. W. Watts and W. R. Burrows, "The Basic Elastic Theory of Vessel Heads under Internal Pressure," J. Appl. Mechanics, Vol 16 (1949), pp 55-73.

2. S. Timoshenko, Theory of Plates and Shells, (New York: McGraw Hill Book Co. Inc., 1940 ).

3. T. Pöschl, Berechnung von Behaltern, (Berlin: Springer Verlag, 2nd ed., 1926).

4. T. Au, E. E. Goodman, and N. M. Newmark, "A Numerical Procedure for the Analysis of Pressure Vessel Heads, "Technical Report, Dept. of Civil Eng., Univ. of Illinois, Urbana Illinois, (Feb. 15, 1951).

5. R. S. Varga, "Numberical Solution of the Two-Group Diffusion Equation in $x-y$ Geometry," IRE Trans. on Nuclear Sci., Vol NS-4 No. 2, (Dec. 1957).

6. D. W. Paceman and H. H. Rachford, Jr., "The Numerical Solution of Parabolic and Elliptic Differential Equations," J. Soc. Ind. Appl. Math., Vol 3 (1955), pp 28-41 .

7. G. Shortley, "Use of Tschebyscheff Polynomial Operators in the Numerical Solution of BoundaryValue Froblems," . J. of Appl. Physics, Vol 24 (1953), pp 392-396.

8. D. Young, "On Richardson's Method for Solving Linear Systems with Positive Definite Matrices," J. of Math. and Phys., Vol 32 (1953), pp 243-255.

9. G. W. Watts and H. A. Lang, "The Stresses in a Pressure Vessel with a. Hemispherical Head," Trans. ASME, Vol 75 (1953), pp 83-89. 